\title{
Expectations in Incremental Discourse Processing
}

\author{
Dan Cristea \\ Faculty of Computer Science \\ University "A.I. Cuza" \\ 16, Berthelot Street \\ 6600 - Iasi, Romania \\ dcristea@infoiasi.ro
}

\author{
Bonnie Webber \\ Dept. of Computer \& Information Science \\ University of Pennsylvania \\ 200 South 33rd Street \\ Philadelphia PA 19104-6389 USA \\ bonnie@central . cis .upenn . edu
}

\begin{abstract}
The way in which discourse features express connections back to the previous discourse has been described in the literature in terms of adjoining at the right frontier of discourse structure. But this does not allow for discourse features that express $e x$ pectations about what is to come in the subsequent discourse. After characterizing these expectations and their distribution in text, we show how an approach that makes use of substitution as well as adjoining on a suitably defined right frontier, can be used to both process expectations and constrain discouse processing in general.
\end{abstract}

\section{Introduction}

Discourse processing subsumes several distinguishable but interlinked processes. These include reference and ellipsis resolution, inference (e.g., inferential processes associated with focus particles such as, in English, "even" and "only"), and identification of those structures underlying a discourse that are associated with coherence relations between its units. In the course of developing an incremental approach to the latter, we noticed a variety of constructions in discourse that raise expectations about its future structural features. We found that we could represent such expectations by adopting a lexical variant of TAG - LTAG (Schabes, 1990) - and using its substitution operation as a complement to adjoining. Perhaps more interesting was that these expectations appeared to constrain the subsequent discourse until they were resolved. This we found we could model in terms of constraints on adjoining and substitution with respect to a suitably defined Right Frontier. This short paper focuesses on the phenomenon of these expectations in discourse and their expression in a discourse-level LTAG. We conclude the paper with some thoughts on incremental discourse processing in light of these expectations.

The following examples illustrate the creation of expectations through discourse markers:

\section{Example 1}

a. On the one hand, John is very generous.

b. On the other, he is extremely difficult to find.

\section{Example 2}

a. On the one hand, John is very generous.

b. On the other, suppose you needed some money.

c. You'd see that he's very difficult to find.

\section{Example 3}

a. On the one hand, John is very generous.

b. For example, suppose you needed some money.

c. You would just have to ask him for it.

b. On the other hand, he is very difficult to find.

Example 1 illustrates the expectation that, following a clause marked "on the one hand", the discourse will express a constrasting situation (here marked by "on the other"). Examples 2 and 3 illustrate that such an expectation need not be satisfied immediately by the next clause: In Example 2, clause (b) partially resolves the expectation set up in (a), but introduces an expectation that the subsequent discourse will indicate what happens in such cases. That expectation is then resolved in clause (c). In Example 3, the next two clauses do nothing to satisfy the expectation raised in clause (a): rather, they give evidence for the claim made in (a). The expectation raised in (a) is not resolved until clause (d).

These examples show expectations raised by sentential adverbs and the imperative use of the verb "suppose". Subordinate conjunctions (e.g., "just as", "although", "when", etc.) can lead to similar expectations when they appear in a preposed subordinate clause - eg.

\section{Example 4}

a. Although John is very generous,

b. if you should need some money,

c. you'd see that he's difficult to find.

As in Example 2, clause 4(a) raises the expectation of learning what is nevertheless the case. Clause 4(b) partially satisfies that expectation by raising a hy- 
pothetical situation, along with the expectation of learning what is true in such a situation. This latter expectation is then satisfied in clause $4(\mathrm{c})$.

In summary, these expectations can be characterized as follows: (1) once raised, an expectation must be resolved, but its resolvant can be a clause that raises its own expectations; (2) a clause raising an expectation can itelf be elaborated before that expectation is resolved, including elaboration by clauses that raise their own expectations; and (3) the most deeply "embedded" expectations must always be resolved first.

Now these are very likely not the only kinds of expectations to be found in discourse: Whenever events or behavior follow fairly regular patterns over time, observers develop expectations about what will come next or at least eventually. For example, a dialogue model may embody the expectation that a suggestion made by one dialogue participant would eventually be followed by an explicit or implicit rejection, acceptance or tabling by the other. Other dialogue actions such as clarifications or justifications may intervene, but there is a sense of an expectation being resolved when the suggestion is responded to.

Here we are focussed on discourse at the level of individual monologue or turn within a larger discourse: what we show is that discourse manifests certain forward-looking patterns that have similar constraints to those of sentence-level syntax and can be handled by similar means. One possible reason that these particualr kinds of expressions may not have been noticed before is that in non-incremental approaches to discourse processing (Mann and Thompson, 1988; Marcu, 1996), they don't stand out as obviously different.

The labels for discourse coherence relations used here are similar to those of RST (Mann and Thompson, 1988), but for simplicity, are treated as binary. Since any multi-branching tree can be converted to a binary tree, no representational power is lost. In doing this, we follow several recent converging computational approaches to discourse analysis, which are also couched in binary terms (Gardent, 1997; Marcu, 1996; Polanyi and van den Berg, 1996; Schilder, 1997; van den Berg, 1996).

Implicit in our discussion is the view that in processing a discourse incrementally, its semantics and pragmatics are computed compositionally from the structure reflected in the coherence relations between its units. In the figures presented here, non-terminal nodes in a discourse structure are labelled with coherence relations merely to indicate the functions that project appropriate content, beliefs and other side effects into the recipient's discourse model. This view is, we believe, consistent with the more detailed formal interfaces to discourse semantics/pragmatics presented in (Gardent, 1997; Schilder, 1997; van den Berg, 1996), and also allows for multiple discourse relations (intentional and in- formational) to hold between discourse units (Moore and Pollack, 1992; Moser and Moore, 1995; Moser and Moore, 1996) and contribute to the semantic/pragmatics effects on the recipient's discourse model.

\section{Expectations in Corpora}

The examples given in the Introduction were all "minimal pairs" created to illustrate the relevant phenomenon as succinctly as possible. Empirical questions thus include: (1) the range of lexicosyntactic constructions that raise expectations with the specific properties mentioned above; (2) the frequency of expectation-raising constructions in text; (3) the frequency with which expectations are satisfied immediately, as opposed to being delayed by material that elaborates the unit raising the expectation; (4) the frequency of embedded expectations; and (5) features that provide evidence for an expectation being satisfied.

While we do not have answers to all these questions, a very preliminary analysis of the Brown Corpus, a corpus of approximately 1600 email messages, and a short Romanian text by $\mathrm{T}$. Vianu (approx. 5000 words) has yielded some interesting results.

First, reviewing the 270 constructions that Knott has identified as potential cue phrases in the Brown Corpus ${ }^{1}$, one finds 15 adverbial phrases (such as "initially", "at first", "to start with", etc.) whose presence in a clause would lead to an expectation being raised. All left-extraposed clauses in English raise expectations (as in Example 4) so all the subordinate conjunctions in Knott's list would be included as well. Outside of cue phrases, we have identified imperative forms of "suppose" and "consider" as raising expectations, but currently lack a more systematic procedure for identifying expectationraising constructions in text than hand-combing text for them.

With respect to how often expectation-raising constructions appear in text, we have Brown Corpus data on two specific types - imperative "suppose" and adverbial "on the one hand" - as well as a detailed analysis of the Romanian text by Vianu mentioned earlier.

There are approximately $54 \mathrm{~K}$ sentences in the Brown Corpus. Of these, 37 contain imperative "suppose" or "let us suppose". Twelve of these correspond to "what if" questions or negotiation moves which do not raise expectations:

Suppose - just suppose this guy was really what he said he was! A retired professional killer If he was just a nut, no harm was done. But if he was the real thing, he could do something about Lolly. (cl23)

\footnotetext{
${ }^{1}$ Personal communication, but also see (Knott, 1996)
} 
Alec leaned on the desk, holding the clerk's eyes with his. "Suppose you tell me the real reason", he drawled. "There might be a story in it". (cl21)

The remaining 25 sentences constitute only about $0.05 \%$ of the Brown Corpus. Of these, 22 have their expectations satisfied immediately $(88 \%)$ - for example,

Suppose John Jones, who, for 1960, filed on the basis of a calendar year, died June 20,1961 . His return for the period January 1 to June 20, 1961, is due April 16, 1962.

One is followed by a single sentence elaborating the original supposition (also flagged by "suppose") -

"Suppose it was not us that killed these aliens. Suppose it is something right on the planet, native to it. I just hope it doesn't work on Earthmen too. These critters went real sudden". (cm04)

while the remaining two contain multi-sentence elaborations of the original supposition. None of the examples in the Brown Corpus contains an embedded expectation.

The adverbial "on the one hand" is used to pose a contrast either phrasally -

Both plans also prohibited common directors, officers, or employees between $\mathrm{Du}$ Pont, Christiana, and Delaware, on the one hand, and General Motors on the other. (ch16)

You couldn't on the one hand decry the arts and at the same time practice them, could you? (ck08)

or clausally. It is only the latter that are of interest from the point of discourse expectations.

The Brown Corpus contains only 7 examples of adverbial "on the one hand". In three cases, the expectation is satisfied immediately by a clause cued by "but" or "or" - e.g.

On the one hand, the Public Health Service declared as recently as October 26 that present radiation levels resulting from the Soviet shots "do not warrant undue public concern" or any action to limit the intake of radioactive substances by individuals or large population groups anywhere in the $\mathrm{Aj}$. But the PHS conceded that the new radioactive particles "will add to the risk of genetic effects in succeeding generations, and possibly to the risk of health damage to some people in the United States".(cb21)

In the remaining four cases, satisfaction of the expectation (the "target" contrast item) is delayed by 2-3 sentences elaborating the "source" contrast item - e.g.
Brooklyn College students have an ambivalent attitude toward their school. On the one hand, there is a sense of not having moved beyond the ambiance of their high school. This is particularly acute for those who attended Midwood High School directly across the street from Brooklyn College. They have a sense of marginality at being denied that special badge of status, the out-of-town school. At the same time, there is a good deal of self-congratulation at attending a good college ...(cf25)

In these cases, the target contrast item is cued by "on the other hand" in three cases and "at the same time" in the case given above. Again, none of the examples contains an embedded expectation.

(The much smaller email corpus contained six examples of clausal "on the one hand", with the target contrast cued by "on the other hand", "on the other" or "at the other extreme". In one case, there was no explicit target contrast and the expectation raised by "on the one hand" was never satisfied. We will continue to monitor for such examples.)

Before concluding with a close analysis of the Romanian text, we should note that in both the Brown Corpus and the email corpus, clausal adverbial "on the other hand" occurs more frequently without an expectation-raising "on the one hand" than it does with one. (Our attention was called to this by a frequency analysis of potential cue phrase instances in the Brown Corpus compiled for us by Alistair Knott and Andrei Mikheev, HCRC, University of Edinburgh.) We found 53 instances of clausal "on the other hand" occuring without an explicit source contrast cued earlier. Although one can only speculate now on the reason for this phenomenon, it does make a difference to incremental analysis, as we try to show in Section 3.3.

The Romanian text that has been closely analysed for explicit expectation-raising constructions is T. Vianu's Aesthetics. It contains 5160 words and 382 discourse units (primarily clauses). Counting preposed gerunds as raising expectations as well as counting the constructions noted previously, 39 instances of expectation-raising discourse units were identified $(10.2 \%)$. In 11 of these cases, $1-16$ discourse units intervened before the raised expectation was satisfied. One example follows:

Dar deşi trebuie să-l parcurgem în intregime, pentru a orienta cercetarea este nevoie să încercăm încă de pe acum o precizare a obiectului lui.

(But although we must cover it entirely, in order to guide the research we need to try already an explanation of its subject matter.) 


\section{A Grammar for Discourse}

The intuitive appeal of Tree-adjoining Grammar (TAG) (Joshi, 1987) for discourse processing (Gardent, 1997; Polanyi and van den Berg, 1996; Schilder, 1997; van den Berg, 1996; Webber, 1991) follows from the fact that TAG's adjoining operation allows one to directly analyse the current discourse unit as a sister to previous discourse material that it stands in a particular relation to. The new intuition presented here - that expectations convey a dependency between the current discourse unit and future discourse material, a dependency that can be "stretched" long-distance by intervening material - more fully exploits TAG's ability to express dependencies. By expressing in an elementary TAG tree, a dependency betwen the current discourse unit and future discourse material and using substitution (Schabes, 1990) when the expected material is found, our TAG-based approach to discourse processing allows expectations to be both raised and resolved.

\subsection{Categories and Operations}

The categories of our TAG-based approach consist of nodes and binary trees. We follow (Gardent, 1997) in associating nodes with feature structures that may hold various sorts of information, including information about the semantic interpretations projected through the nodes, constraints on the specific operations a node may participate in, etc. A nonterminal node represents a discourse relation holding between its two daughter nodes. A terminal node can be either non-empty (Figure 1a), corresponding to a basic discourse unit (usually a clause), or empty. A node is "empty" only in not having an associated discourse unit or relation: it can still have an associated feature structure. Empty nodes play a role in adjoining and substitution, as explained below, and hence in building the derived binary tree that represents the structure of the discourse.

Adjoining adds to the discourse structure an auxiliary tree consisting of a root labelled with a discourse relation, an empty foot node (labelled ${ }^{*}$ ), and at least one non-empty node (Figures $1 \mathrm{c}$ and $1 \mathrm{~d}$ ). In our approach, the foot node of an auxiliary tree must be its leftmost terminal because all adjoining operations take place on a suitably defined right frontier (i.e., the path from the root of a tree to its rightmost leaf node) - such that all newly introduced material lies to the right of the adjunction site. (This is discussed in Section 3.2 in more detail.) Adjoining corresponds to identifying a discourse relation between the new material and material in the previous discourse that is still open for elaboration.

Figure 2(a) illustrates adjoining midway down the RF of tree $\alpha$, while Figure 2(b) illustrates adjoining at the root of $\alpha$ 's RF. Figure 2(c) shows adjoining at the "degenerate" case of a tree that consists only of its root. Figure 2(d) will be explained shortly.

Substitution unifies the root of a substitution structure with an empty node in the discourse tree that serves as a substitution site. We currently use two kinds of substitution structures: non-empty nodes (Figure 1a) and elementary trees with substitution sites (Figure 1b). The latter are one way by which a substitution site may be introduced into a tree. As will be argued shortly, substitution sites can only appear on the right of an elementary tree, although any number of them may appear there (Figure $1 \mathrm{~b})$. Figure 2(e) illustrates substitution of a nonempty node at $\downarrow$, and Figure 2(f) illustrates substitution of an elementary tree with its own substitution site at $\downarrow_{1}$

Since in a clause with two discourse markers (as in Example 3b) one may look backwards ("for example") while the other looks forwards ("suppose"), we also need a way of introducing expectations in the context of adjoining. This we do by allowing an $a u x-$ iliary tree to contain substitution sites (Figure 1d) which, as above, can only appear on its right. ${ }^{2}$ Another term we use for auxiliary trees is adjoining structures.

\subsection{Constraints}

Earlier we noted that in a discourse structure with no substitution sites, adjoining is limited to the right frontier (RF). This is true of all existing TAG-based approaches to discourse processing (Gardent, 1997; Hinrichs and Polanyi, 1986; Polanyi and van den Berg, 1996; Schilder, 1997; Webber, 1991), whose structures correspond to trees that lack substitution sites. One reason for this RF restriction is to maintain a strict correspondence between a left-to-right reading of the terminal nodes of a discourse structure and the text it analyses - i.e.,

Principle of Sequentiality: A left-toright reading of the terminal frontier of the tree associated with a discourse must correspond to the span of text it analyses in that same left-to-right order.

Formal proof that this principle leads to the restriction of adjoining to the right frontier is given in (Cristea and Webber, June 1997).

The Principle of Sequentiality leads to additional constraints on where adjoining and substitution can occur in trees with substitution sites. Consider the tree in Figure 3(i), which has two such sites, and an adjoining operation on the right frontier at node $\mathrm{R}_{j}$ or above. Figure 3(ii) shows that this would introduce a non-empty node $\left(\mathrm{u}_{k}\right)$ above and to the right of the substitution sites. This would mean that later substitution at either of them would lead to a violation of the Principle of Sequentiality, since the newly

\footnotetext{
${ }^{2}$ We currently have no linguistic evidence for the structure labelled $\beta$ in Figure 1d, but are open to its possibility.
} 


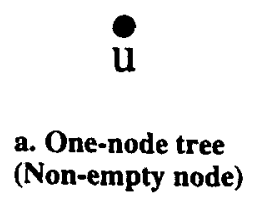

(Non-empty node)

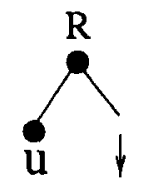

b. Elementary trees with substitution sites

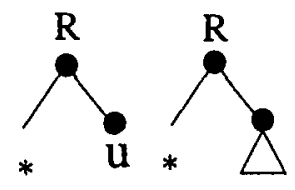

c. Auxiliary trees

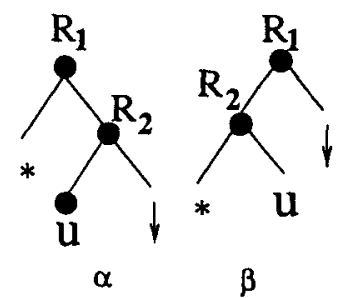

d. Aux trees with substitution sites

Figure 1: Grammatical Categories. (* marks the foot of an auxiliary tree, and $\downarrow$, a substitution site.)

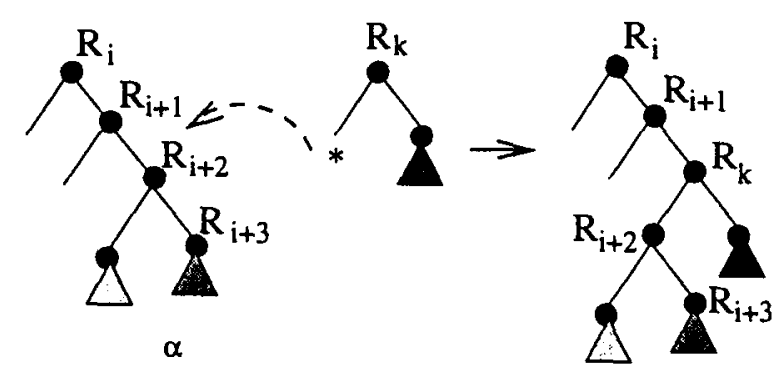

(a) Adjoining at $\mathrm{Ri}+2$ on the RF of $\alpha$

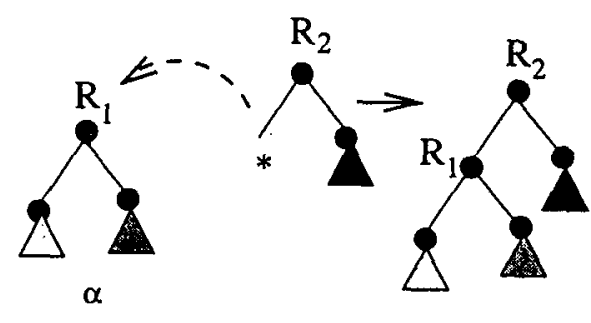

(b) Adjoining at the root (R1) of $\alpha$

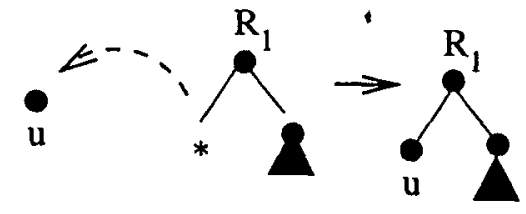

(c) Adjoining at root of single node tree $\alpha$

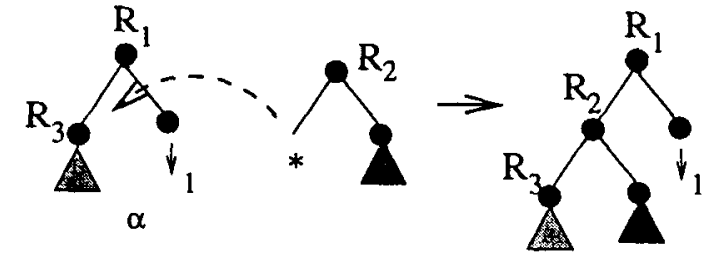

(d) Adjoining at R3 on the right frontier of $\alpha$

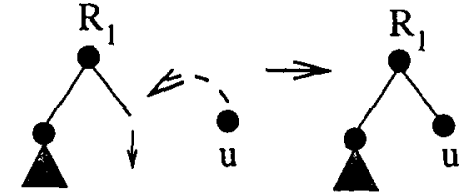

(e) Substituting a material node at

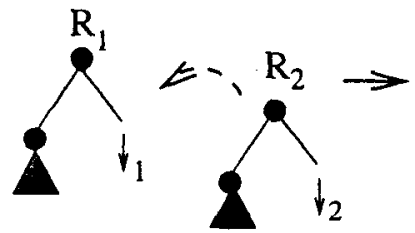

(f) Substituting at $\downarrow_{\text {pn elementary }}$

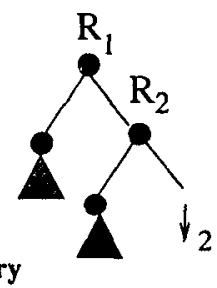
tree with substitution site $\downarrow_{2}$

Figure 2: Examples of Adjoining and Substitution 


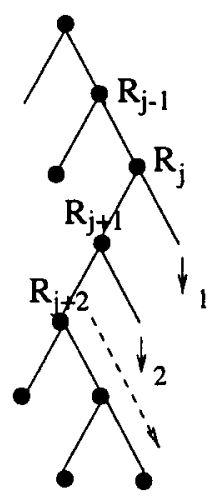

(i)

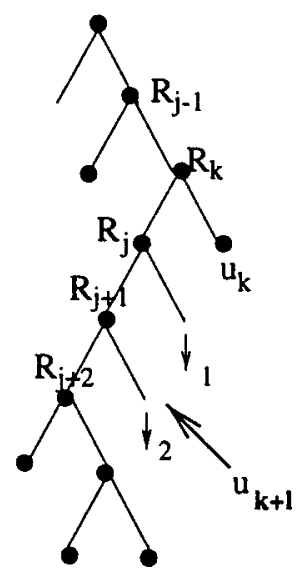

(ii)

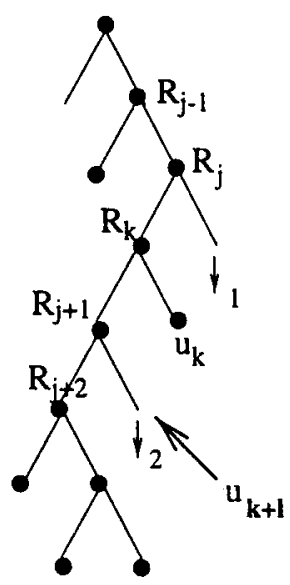

(iii)

Figure 3: Adjoining is constrained to nodes the inner-RF, indicated by the dashed arrow.

substituted node $\mathrm{u}_{k+l}$ would then appear to the left of $\mathrm{u}_{k}$ in the terminal frontier, but to the right of it in the original discourse. Adjoining at any node above $\mathrm{R}_{j+2}$ - the left sister of the most deeply embedded substitution site - leads to the same problem (Figure 3iii). Thus in a tree with substitution sites, adjoining must be limited to nodes on the path from the left sister of the most embedded site to that sister's rightmost descendent. But this is just a right frontier (RF) rooted at that left sister. Thus, adjoining is always limited to a RF: the presence of a substitution site just changes what node that RF is rooted at. We can call a RF rooted at the left sister of the most embedded substitution site, the inner right frontier or "inner_RF". (In Figure 3(i), the inner-RF is indicated by a dashed arrow.) In contrast, we sometimes call the $R F$ of a tree without substitution sites, the outer right frontier or "outer_RF". Figure 2(d) illustrates adjoining on the inner_RF of $\alpha$, a tree with a substitution site labelled $\downarrow_{1}$.

Another consequence of the Principle of Sequentiality is that the only node at which substitution is allowed in a tree with substitution sites is at the most embedded one. Any other substitution would violate the principle. (Formal proof of these claims are given in (Cristea and Webber, June 1997).

\subsection{Examples}

Because we have not yet implemented a parser that embodies the ideas presented so far, we give here an idealized analysis of Examples 2 and 3, to show how an ideal incremental monotonic algorithm that admitted expectations would work.

Figure 4A illustrates the incremental analysis of Example 2. Figure 4A(i) shows the elementary tree corresponding to sentence $2 \mathrm{a}$ ("On the one hand ..."): the interpretation of "John is very generous" corresponds to the left daughter labelled "a". The adverbial "On the one hand" is taken as signalling a coherence relation of Contrast with something expected later in the discourse.

In sentence $2 \mathrm{~b}$ ("On the other hand, suppose ..."), the adverbial "On the other hand" signals the expected contrast item. Because it is already expected, the adverbial does not lead to the creation of a separate elementary tree (but see the next example). The imperative verb "suppose", however, signals a coherence relation of antecedent/consequent $(\mathrm{A} / \mathrm{C})$ with a consequence expected later in the discourse. The elementary tree corresponding to "suppose ..." is shown in Figure $4 \mathrm{~A}$ (ii), with the interpretation of "you need money" corresponding to the left daughter labelled "b". Figure 4A(iii) shows this elementary tree substituted at $\downarrow_{1}$, satisfying that expectation. Figure $4 \mathrm{~A}$ (iv) shows the interpretation of sentence $2 \mathrm{c}$ ("You'd see he's very difficult to find") substituted at $\downarrow_{2}$, satisfying that remaining expectation.

Before moving on to Example 3, notice that if Sentence $2 \mathrm{a}$ were not explicitly cued with "On the other hand", the analysis would proceed somewhat differently.

\section{Example 5}

a. John is very generous.

b. On the other hand, suppose you needed money.

c. You'd see that he's very difficult to find.

Here, the interpretation of sentence 5(a) would correspond to the degenerate case of a tree consisting of a single non-empty node shown in Figure $4 \mathrm{~B}(\mathrm{i})$. The contrast introduced by "On the other hand" in sentence 5(b) leads to the auxiliary tree shown in Figure $4 \mathrm{~B}$ (ii), where $\mathrm{T}$ stands for the elementary tree corresponding to the interpretation of "suppose ...". 


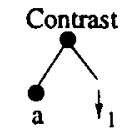

(i)

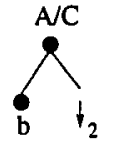

(ii)

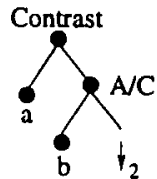

(iii)

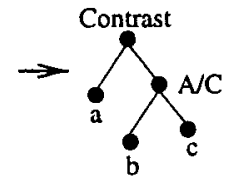

(iv)

A. Example 2

B. Example 5

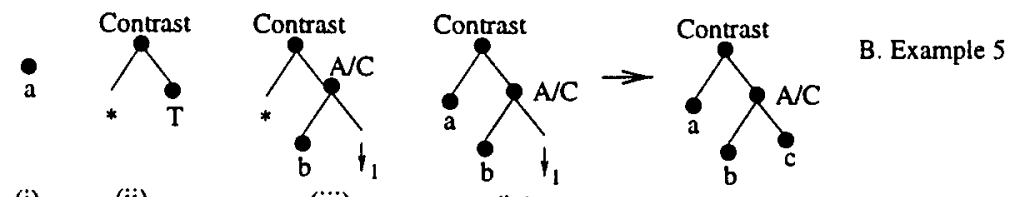

(i)

(ii)

(iii)

(iv)

(v)

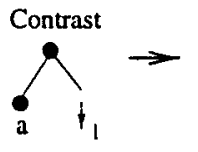

(i)

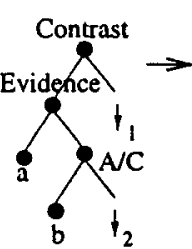

(ii)

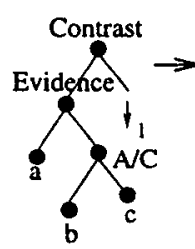

(iii)

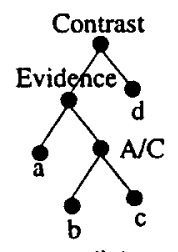

(iv)

Figure 4: Analyses of Examples 2, 3 and 4.

The entire structure associated with sentence $5(\mathrm{~b})$ is shown in Figure $4 \mathrm{~B}(\mathrm{iii})$. This is adjoined to the single node tree in Figure 4B(i), yielding the tree shown in Figure $4 \mathrm{~B}(\mathrm{iv})$. The analysis then continues exactly as in that of Example 2 above.

Moving on to Example 3, Figure $4 \mathrm{C}(\mathrm{i})$ shows the same elementary tree as in Figure 4A(i) corresponding to clause 3a. Next, Figure $4 \mathrm{C}$ (ii) shows the auxiliary tree with substitution site $\downarrow_{2}$ corresponding to clause $3 \mathrm{~b}$ being adjoined as a sister to the interpretation of clause $3 \mathrm{a}$, as evidence for the claim made there. The right daughter of the node labelled "Evidence" is, as in Example 2b, an elementary tree expecting the consequence of the supposition "you need money". Figure 4C(iii) shows the interpretation of clause $3 c$ substituted at $\downarrow_{2}$, satisfying that expectation. Finally, Figure $4 \mathrm{C}(\mathrm{iv})$ shows the interpretation of clause $3 \mathrm{~d}$ substituted at $\downarrow_{1}$, satisfying the remaining expectation.

\section{Sources of Uncertainty}

The idealized analysis presented above could lead to a simple deterministic incremental algorithm, if there were no uncertainty due to local or global ambiguity. But there is. We can identify three separate sources of uncertainty that would affect incremental processing according to the grammar just presented:

- the identity of the discourse relation that is meant to hold between two discourse units;
- the operation (adjoining or substitution) to be used in adding one discourse unit onto another;

- if that operation is adjoining, the site in the target unit at which the operation should take place - that is, the other argument to the discourse relation associated with the root of the auxiliary tree.

It may not be obvious that there could be uncertainty as to whether the current discourse unit satisfies an expectation and therefore substitutes into the discourse structure, or elaborates something in the previous discourse, and therefore adjoins into it. ${ }^{3}$ But the evidence clarifying this local ambiguity may not be available until later in the discourse. In the following variation of Example 4, the fact that clause (b) participates in elaborating the interpretation of clause (a) rather than in satisfying the expectation it raises (which it does in Example 4) may not be unambiguously clear until the discourse marker "for example" in clause (c) is processed.

\section{Example 6}

a. Because John is such a generous man-

b. whenever he is asked for money,

c. he will give whatever he has, for example -

d. he deserves the "Citizen of the Year" award.

The other point is that, even if a forward-looking cue phrase signals only a substitution structure as

\footnotetext{
${ }^{3}$ This is not the same as shift-reduce uncertainty.
} 
in Figure $4 \mathrm{~A}(\mathrm{i})$ and $4 \mathrm{~A}(\mathrm{ii})$, if there are no pending subsitution sites such as $\downarrow_{1}$ in $4 \mathrm{~A}$ (i) against which to unify such a structure, then the substitution structure must be coerced to an auxiliary tree as in Figure 1d (with some as yet unspecified cohesion relation) in order to adjoin it somewhere in the current discourse structure.

\section{Speculations and Conclusions}

In this paper, we have focussed on discourse expectations associated with forward-looking clausal connectives, sentential adverbs and the imperative verbs ("suppose" and "consider"). There is clearly more to be done, including a more complete characterization of the phenomenon and development of an incremental discourse processor based on the ideas presented above. The latter would, we believe, have to be coupled with incremental sentence-level processing. As the previous examples have shown, the same phenomenon that occurs inter-sententially in Examples 1-3 occurs intra-sententially in Examples 4 and 6 , suggesting that the two processors may be based on identical principles. In addition, carrying out sentence-level processing in parallel with discourse processing and allowing each to inform the other would allow co-reference interpretation to follow from decisions about discourse relations and vice versa.

\section{Acknowledgements}

Support for this work has come from the Department of Computer Science, Universiti Sains Malaysia (Penang, Malaysia), the Department of Computer Science, University "A.I.Cuza" (Iasi, Romania) and the Advanced Research Project Agency (ARPA) under grant N6600194C6-043 and the Army Research Organization (ARO) under grant DAAHO494GO426. Thanks go to both the anonymous reviewers and the following colleagues for their helpful comments: Michael Collins, Claire Gardent, Udo Hahn, Joseph Rosenzweig, Donia Scott, Mark Steedman, Matthew Stone, Michael Strube, and Michael Zock. Thanks also to Alistair Knott and Andrei Mikheev for giving us a rough count of cue phrases in the Brown Corpus.

\section{References}

Cristea, Dan and Bonnie Webber. June 1997. Expectations in incremental discourse processing. Technical report, University A.I. Cuza, Iasi, Romania.

Gardent, Claire. 1997. Discourse tree adjoining grammars. Claus report nr.89, University of the Saarland, Saarbrücken.
Hinrichs, Erhard and Livia Polanyi. 1986. Pointing the way: A unified treatment of referential gesture in interactive discourse. In CLS 22, Part 2: Papers from the Parasession on Pragmatics and Grammatical Theory, pages 298-314, Chicago Linguistic Society.

Joshi, Aravind. 1987. An introduction to Tree Adjoining Grammar. In Alexis Manaster-Ramer, editor, Mathematics of Language. John Benjamins, Amsterdam.

Knott, Alistair. 1996. A Data-driven Methodology for Motivating a Set of Coherence Relations. Ph.D. thesis, Department of Artificial Intelligence, University of Edinburgh.

Mann, William and Sandra Thompson. 1988. Rhetorical structure theory: Toward a functional theory of text organization. Text, 8(3):243-281.

Marcu, Daniel. 1996. Building up rhetorical structure trees. In Proceedings of $A A A I-96$, pages 1069-1074, Portland OR.

Moore, Johanna and Martha Pollack. 1992. A problem for rst: The need for multi-level discouse analysis. Computational Linguistics, 18(4):537-544.

Moser, Megan and Johanna Moore. 1995. Investigating cue selection and placement in tutorial discourse. In Proc. 33rd Annual Meeting, Association for Computational Linguistics, pages 130135, MIT, Boston MA.

Moser, Megan and Johanna Moore. 1996. Toward a synthesis of two accounts of discourse structure. Computational Linguistics, 22(2):TBA.

Polanyi, Livia and Martin H. van den Berg. 1996. Discourse structure and discourse interpretation. In P. Dekker and M. Stokhof, editors, Proceedings of the Tenth Amsterdam Colloquium, pages 113131, ILLC/Department of Philosophy, University of Amsterdam.

Schabes, Yves. 1990. Mathematical and Computational Aspects of Lexicalized Grammars. Ph.D. thesis, Department of Computer and Information Science, University of Pennsylvania. Technical Report MS-CIS-90-48, LINC Lab 179.

Schilder, Frank. 1997. Tree discourse grammar, or how to get attached to a discourse. In Proceedings of the Tilburg Conference on Formal Semantics, Tilburg, Netherlands, January.

van den Berg, Martin H. 1996. Discourse grammar and dynamic logic. In P. Dekker and M. Stokhof, editors, Proceedings of the Tenth Amsterdam Colloquium, pages 93-111, ILLC/Department of Philosophy, University of Amsterdam.

Webber, Bonnie. 1991. Structure and ostension in the interpretation of discourse deixis. Natural Language and Cognitive Processes, 6(2):107-135. 